\title{
Monotonicity and complete monotonicity for continuous-time Markov chains
}

\author{
Monotonie et monotonie complète \\ des chaînes de Markov à temps continu \\ Paolo Dai Pra ${ }^{\mathrm{a}}$, Pierre-Yves Louis ${ }^{\mathrm{b}}$, Ida Minelli ${ }^{\mathrm{a}}$ \\ ${ }^{a}$ Dipartimento di Matematica Pura e Applicata, Università di Padova, Via Belzoni 7, 35131 Padova, Italy \\ ${ }^{\mathrm{b}}$ Institut für Mathematik, Potsdam Universität, Am neuen Palais, 10 - Sans Souci, D-14 415 Potsdam, Germany \\ http://www.sciencedirect.com/science/article/pii/S1631073X06001646 \\ Post-print of Comptes Rendus Mathematique, \\ Volume 342, Issue 12, 15 June 2006, pp. 965-970, ISSN 1631-073X \\ doi:10.1016/j.crma.2006.04.007 \\ Creative Commons Attribution Non-Commercial No Derivatives License
}

\begin{abstract}
We analyze the notions of monotonicity and complete monotonicity for Markov Chains in continuous-time, taking values in a finite partially ordered set. Similarly to what happens in discrete-time, the two notions are not equivalent. However, we show that there are partially ordered sets for which monotonicity and complete monotonicity coincide in continuous time but not in discrete-time. To cite this article: P. Dai Pra et al., C. R. Acad. Sci. Paris, Ser. I 342 (2006).
\end{abstract}

Résumé

Nous étudions les notions de monotonie et de monotonie complète pour les processus de Markov (ou chaînes de Markov à temps continu) prenant leurs valeurs dans un espace partiellement ordonné. Ces deux notions ne sont pas équivalentes, comme c'est le cas lorsque le temps est discret. Cependant, nous établissons que pour certains ensembles partiellement ordonnés, l'équivalence a lieu en temps continu bien que n'étant pas vraie en temps discret. Pour citer cet article :P. Dai Pra et al., C. R. Acad. Sci. Paris, Ser. I 342 (2006)

Email addresses: daipra@math.unipd.it (Paolo Dai Pra), louis@math.uni-potsdam.de (Pierre-Yves Louis), minelli@math. unipd.it (Ida Minelli). 


\section{Version française abrégée}

L'utilisation des chaînes de Markov dans le cadre des algorithmes MCMC soulève de nombreuses questions au sein desquelles la monotonie joue un rôle important. Deux notions de monotonie sont considérées pour les chaînes de Markov à valeurs dans un espace partiellement ordonné $(S,<)$ (poset selon la terminologie anglaise). Nous supposons $S$ fini et les chaînes (resp. processus) de Markov homogènes en temps. Dans la définition 1.1 nous reformulons la notion de monotonie, équivalente à la définition usuelle via la propriété de stabilité des fonctions croissantes $f$ sous l'action de l'opérateur de transition $T_{t} f(y):=$ $\sum_{x \in S} f(x) P_{t}(x, y)$.

Pour différentes applications et en particulier à des fins de simulation, une notion plus forte, dite de monotonie complète a été introduite dans la Définition 1.2. En d'autres termes, cette monotonie complète signifie que l'on peut coupler simultanément tous les processus partant de toutes les conditions initiales possibles, en préservant l'ordre entre ces processus. Cette propriété s'avère essentielle pour l'utilisation de l'algorithme de Propp \& Wilson (cf. [7]).

Aucune méthode générale n'existe pour caractériser simplement la monotonie complète à partir des probabilités de transition ou du générateur infinitésimal. De manière évidente, pour une chaîne (resp. processus ) de Markov donnée à valeurs dans $S$, la monotonie complète implique la monotonie. La question est alors de caractériser les posets $S$ pour lesquels l'implication réciproque est vraie pour tout processus. Pour les chaînes de Markov, i.e. lorsque le temps est discret, ce problème a été résolu dans [3] (cf. Theorem 1.3 de la section 1). Dans cette note nous considérons cette question pour des processus de Markov (ou chaînes de Markov à temps continu) réguliers.

Soient $L=\left(L_{x, y}\right)_{x, y \in S}$ le générateur infinitésimal d'un processus de Markov régulier et $S_{2}:=S \times S \backslash\{(x, x): x \in S\}$. Le générateur $L$ peut être identifié à un élément du cône $\left(\mathbb{R}^{+}\right)^{S_{2}}$.

Notre technique principale consiste à caractériser l'ensemble des générateurs monotones $\mathcal{G}_{m o n}$ et celui des générateurs complètement monotones $\mathcal{G}_{\text {c.mon }}$ comme des cônes de $\left(\mathbb{R}^{+}\right)^{S_{2}}$.

La monotonie d'un générateur $L$ est reformulée de manière géométrique (2) dans la proposition $\mathbf{2 . 2}$ en fonction des vecteurs $W^{\Gamma, x, y} \in \mathbb{R}^{S_{2}}$ (définis par (1)) où $x, y \in S, \Gamma$ up-set (cf. Définition 2.1). La représentation (2) du cône $\mathcal{G}_{\text {mon }}$ comme intersection de demi-espaces est appelée $H$-représentation (cf. [4]).

Dans la proposition 2.3 la monotonie complète d'un générateur $L$ est caractérisée par sa décomposition (4) en tant que combinaison linéraire à coefficients positifs des vecteurs $\mathbb{I}_{f} \in\left(\mathbb{R}^{+}\right)^{S_{2}}$ définis en (3) où $f \in \mathcal{M}$, i.e. où $f$ est une fonction croissante de $S$ dans $S$. La représentation (4) du cône $\mathcal{G}_{c . m o n}$ via les rayons extrémaux $\mathbb{I}_{f}$ est appelée $V$-représentation.

Puisque, pour toute fonction $f$ croissante, $\Gamma$ up-set, $x, y \in S$, on a $\left\langle\mathbb{I}_{f}, W^{\Gamma, x, y}\right\rangle \geq 0$, on a bien l'inclusion $\mathcal{G}_{c . m o n} \subseteq \mathcal{G}_{\text {mon }}$. Il apparaît dans un premier temps (Proposition 2.4) que si l'équivalence entre les deux notions de monotonie a lieu pour toute chaîne de Markov à valeurs dans un poset $S$, alors cette équivalence demeure pour le poset $S$ lorsque l'on considère des processus à temps continu. La réciproque s'avère cependant fausse comme les résultats ci-après le prouve.

Nous donnons la liste complète des posets d'au plus cinq élements pour lesquels l'équivalence est fausse. Nous n'avons malheureusement pas réussi à caractériser tous les posets pour lesquels l'équivalence entre les deux notions de monotonie est vérifiée, i.e. pour lesquels $\mathcal{G}_{c . m o n}=\mathcal{G}_{m o n}$ a lieu. Nous prouvons :

Proposition 3.1 Les seuls posets $S$ avec $\sharp S \leq 5$ tels que les deux notions de monotonie ne sont pas équivalentes sont ceux dont le diagramme de Hasse (cf. définition in section 2 [3], p. 943) est représenté dans la figure 1.

En particulier, cela signifie que $\mathcal{G}_{m o n}=\mathcal{G}_{c . m o n}$ pour les deux posets importants de cardinal quatre, à savoir le diamant et le noeud-papillon, dont les diagrammes de Hasse sont respectivement, dans la figure 1, $\mathcal{S}_{1}$ et $\mathcal{S}_{4}$ sans l'élément $w$. Remarquons que l'équivalence est fausse pour les chaînes de Markov à valeurs 
dans ces deux posets (cf. les exemples 1.1 et 4.5 de [3]).

Bien plus, nous établissons la Proposition suivante qui se fonde sur une technique permettant de 《relever» les générateurs monotones non complétement monotones à valeurs dans un poset $S^{\prime}$ (par exemple de la figure 1) en un générateur monotone non complétement monotone à valeurs dans un poset $S$ contenant $S^{\prime}$.

Proposition 3.3 Si un poset $S$ admet comme sous-poset (cf. Définition 3.2) un poset $S^{\prime}$ dont le diagramme de Hasse est représenté dans les figures 1 et 2, alors les deux notions de monotonie ne sont pas équivalentes sur $S$.

Grâce à ce résultat et à des calculs directs, nous établissons également une liste exhaustive des posets de cardinal six où l'équivalence n'est pas vérifiée. Nous renvoyons à la publication [2] pour plus de détails. La figure 2 donne les exemples caractéristiques de cardinal six pour lesquels l'équivalence est fausse.

\section{Introduction}

The use of Markov chains in simulation has raised a number of questions concerning qualitative and quantitative features of random processes, in particular in connection with mixing properties. Among the features that are useful in the analysis of effectiveness of Markov Chain Monte Carlo (MCMC) algorithms, a relevant role is played by monotonicity. Two notions of monotonicity have been proposed, for Markov chains taking values in a partially ordered set $S$ (poset from now on). To avoid measurability issues, that are not relevant for our purposes, we shall always assume $S$ to be finite. Moreover, all Markov chains are implicitely assumed to be time-homogeneous.

Definition 1.1 A Markov chain $\left(\eta_{t}\right), t \in \mathbb{R}^{+}$or $t \in \mathbb{Z}^{+}$, on the poset $S$, with transition probabilities $P_{t}(x, y):=P\left(\eta_{t}=x \mid \eta_{0}=y\right)$, is said to be monotone if for each pair $y, z \in S$ with $y \leq z$ there exists a Markov chain $\left(X_{t}(y, z)\right)$ on $S \times S$ such that

(i) $X_{0}(y, z)=(y, z)$ a.s.

(ii) Each component $\left(X_{t}^{i}(y, z)\right), i=1,2$ is a Markov chain on $S$ with transition probabilities $P_{t}(x, y)$.

(iii) for all $t \geq 0, X_{t}^{1}(y, z) \leq X_{t}^{2}(y, z)$ a.s.

There are various equivalent formulation of monotonicity. For instance, defining the transition operator $T_{t} f(y):=\sum_{x \in S} f(x) P_{t}(x, y)$, then the chain is monotone if and only if $T_{t}$ maps increasing functions into increasing functions. This characterization can be turned (see Proposition 2.2) into a simple algorithm for checking monotonicity of Markov chains in terms of the element of the transition matrix (in discrete-time) or in terms of the infinitesimal generator (in continuous-time).

For various purposes, including simulation, a stronger notion of monotonicity has been introduced.

Definition 1.2 A Markov chain $\left(\eta_{t}\right), t \in \mathbb{R}^{+}$or $t \in \mathbb{Z}^{+}$, on the poset $S$, with transition probabilities $P_{t}(x, y):=P\left(\eta_{t}=x \mid \eta_{0}=y\right)$, is said to be completely monotone if there exists a Markov chain $\left(\xi_{t}(\cdot)\right)$ on $S^{S}$ such that

(i) $\xi_{0}(y)=y$ a.s.

(ii) For every fixed $z \in S$, the process $\left(\xi_{t}(z)\right)$ is a Markov chain with transition probabilities $P_{t}(x, y)$.

(iii) If $y \leq z$, then for every $t \geq 0$ we have $\xi_{t}(y) \leq \xi_{t}(z)$ a.s.

In other words, complete monotonicity means that we can simultaneously couple in an order preserving way all processes leaving any possible initial state. This property becomes relevant when one aims at sampling from the stationary measure of a Markov chain using the Propp \& Wilson algorithm (see [7]). If the transition probabilities, or the infinitesimal generator, are known, no simple general rule for checking complete monotonicity is known. Since, obviously, complete monotonicity implies monotonicity, a natural 
question is to determine for which posets the converse is true. This problem has been completely solved in [3] for discrete-time Markov chain: in this case the following result holds.

Theorem 1.3 Every monotone Markov chain in the poset $S$ is also completely monotone if and only if $S$ is acyclic, i.e. there is no loop $x_{0}, x_{1}, \ldots, x_{n}, x_{n+1}=x_{0}$ such that, for $i=0,1, \ldots, n$

(i) $\forall j=0,1, \ldots, n, j \neq i, x_{i} \neq x_{j}$,

(ii) Either $x_{i}<x_{i+1}$ or $x_{i}>x_{i+1}$,

(iii) $x_{i} \leq y \leq x_{i+1}$ or $x_{i} \geq y \geq x_{i+1}$ implies $y=x_{i}$ or $y=x_{i+1}$.

Our aim in this paper is to deal with the same problem in continuous-time, for regular Markov chains, i.e. Markov chains possessing an infinitesimal generator (or, equivalently, jumping a.s. finitely many times in any bounded time interval). We have not been able to provide a complete link between discrete and continuous-time. It turns out that if in a poset $S$ monotonicity implies complete monotonicity in discrete-time, then the same holds true in continous-time (see Proposition 2.4). The converse is not true, however; in the two four-points cyclic posets (the diamond and the bowtie, following the terminology in [3]) equivalence between monotonicity and complete monotonicity holds in continuous but not in discrete-time. There are, however, five-points posets in which equivalence fails in continuous-time as well.

In this paper we do not achieve the goal of characterizing all posets for which equivalence holds. Via a computer-aided (but exact) method we give a complete list of five and six point posets for which equivalence fails. Moreover we show that in each poset containing one of the former as subposet, equivalence fails as well (this does not follow in a trivial way).

In Section 2 we give some preliminary notions, whose aim is to put the complete monotonicity problem in continuous-time on a firm basis. Our main results are given in Section 3. All details not contained in this note will be given in the forthcoming paper [2].

\section{Preliminaries}

Let $(S,<)$ be a finite poset, and $L=\left(L_{x, y}\right)_{x, y \in S}$ be the infinitesimal generator of a regular Markov chain. Let $S_{2}:=S \times S \backslash\{(x, x): x \in S\}$. An infinitesimal generator is a matrix $L=\left(L_{x, y}\right)_{x, y \in S}$ whose nondiagonal elements are nonnegative, while the terms in the diagonal are given by $L_{x, x}=-\sum_{y \neq x} L_{x, y}$. Thus $L$ may be identified with an element of the cone $\left(\mathbb{R}^{+}\right)^{S_{2}}$. Our main tool for understanding the relations between the two notions of monotonicity consists in representing the sets of generators of monotone and completely monotone Markov chains as subcones of $\left(\mathbb{R}^{+}\right)^{S_{2}}$.

Definition 2.1 A subset $\Gamma \subseteq S$ is said to be an up-set if $x \in \Gamma$ and $x \leq y \Rightarrow y \in \Gamma$.

The following proposition is a reformulation of a result in $[6]$. By $\langle\cdot, \cdot\rangle$ we denote the Euclidean scalar product in $(\mathbb{R})^{S_{2}}$.

Proposition 2.2 For given $x, y \in S, \Gamma$ up-set, let $W^{\Gamma, x, y} \in \mathbb{R}^{S_{2}}$ be defined by

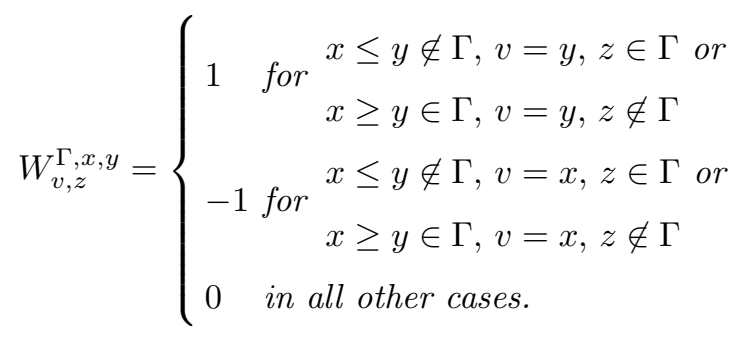

Then the set $\mathcal{G}_{\text {mon }}$ of generators of monotone Markov chains is the subcone of the elements $L \in\left(\mathbb{R}^{+}\right)^{S_{2}}$ satisfying the inequalities 


$$
\left\langle L, W^{\Gamma, x, y}\right\rangle \geq 0 \text { for every } \Gamma, x, y .
$$

Let now $\mathcal{M}$ denote the set of increasing functions $S \rightarrow S$. For $f \in \mathcal{M}$, let $\mathbb{I}_{f} \in\left(\mathbb{R}^{+}\right)^{S_{2}}$ be defined by

$$
\left(\mathbb{I}_{f}\right)_{x, y}=\left\{\begin{array}{l}
1 \text { if } f(x)=y \\
0 \text { otherwise }
\end{array}\right.
$$

Our first result is the following description of the set of generators of completely monotone Markov chains. Its proof is a rather straightforward consequence of the existence of the completely monotone coupling (the process $\left(\xi_{t}\right)$ in Definition 1.2).

Proposition 2.3 The set $\mathcal{G}_{c . m o n}$ of generators of completely monotone Markov chains is the cone given by linear combination with nonnegative coefficients of the vectors $\mathbb{I}_{f}$, i.e.

$$
L=\sum_{f \in \mathcal{M}} \Lambda_{f} \mathbb{I}_{f}, \text { with } \Lambda_{f} \geq 0 .
$$

Note that, for each $f \in \mathcal{M}, \Gamma$ up-set, $x, y \in S$, we have $\left\langle\mathbb{I}_{f}, W^{\Gamma, x, y}\right\rangle \geq 0$, i.e. we recover the inclusion $\mathcal{G}_{\text {c.mon }} \subseteq \mathcal{G}_{\text {mon }}$. Our aim is to determine for which posets the converse inclusion holds true.

A partial result in this direction is given by the following proposition, which establishes a comparison with the discrete-time case. Its proof relies on analogous representations in terms of cones for discrete-time transition matrix.

Proposition 2.4 Suppose that in the poset $S$ monotonicity and complete monotonicity are equivalent notions for discrete-time Markov chains. Then the equivalence holds in continuous-time as well.

\section{Extremal generators of monotone Markov chains: the monotonicity equivalence for "small" posets and extension to larger posets}

According to the previous subsection, the equivalence between the complete monotonicity and the monotonicity of any Markov Chain on a poset $S$ is equivalent to

$$
\mathcal{G}_{c . m o n}=\mathcal{G}_{m o n} .
$$

In this section we answer the question for posets with a small cardinality.

First note that the cases $\sharp S=2, \sharp S=3$ are obvious: in these cases $S$ is linearly totally ordered (following the terminology of [3]). According to Theorem 1.3, there is equivalence for all the discrete-time Markov chains and using the result of Proposition 2.4 the equivalence holds for continuous-time Markov chains as well.

In order to further investigate the equality (5) we developed computer computations. The cone $\mathcal{G}_{\text {mon }}$ is defined as intersection of half spaces in (2) (so called $H$-representation). The cone $\mathcal{G}_{c . m o n}$ is defined by its extremal rays in (4) (so called V-representation). The software $c d d+$ (see [1]) is able to compute exactly one representation given the other one. This is a $C++$ implementation for convex polyhedron of the Double Description Method (see for instance [4]). Finding the extremal rays of the cone $\mathcal{G}_{\text {mon }}$ and the (minimal) set of inequalities defining the cone $\mathcal{G}_{c . m o n}$, we are then able to know if $\mathcal{G}_{\text {mon }} \subseteq \mathcal{G}_{c . m o n}$ holds.

We operated by first using the software GAP (see [5]) in order to

(i) find the up-sets $\Gamma$ related to the poset $S$, the vectors $W^{\Gamma, x, y} \in \mathbb{R}^{S_{2}}$ and then identify the H-representation of $\mathcal{G}_{\text {mon }}$;

(ii) compute all the increasing functions $f \in \mathcal{M}$, identify the vectors $\mathbb{I}_{f} \in\left(\mathbb{R}^{+}\right)^{S_{2}}$ and then find the V-representation of $\mathcal{G}_{\text {c.mon }}$. 
We then use the software $c d d+$ to produce the other representations of the cones, and the software Scilab (see [8]) to test if $\mathcal{G}_{\text {mon }} \subseteq \mathcal{G}_{\text {c.mon }}$.

The difficulty in applying this method to posets with a high cardinality relies on the combinatorial complexity of the step (ii) and on the time the software $c d d+$ needs. Rather than to $\sharp S$, this time is related to the number of facets of the cones, which comes from the partial order structure. Nevertheless, we were able to completely study the cases when $\sharp S \leq 6$.

Here we briefly give the cases $\sharp S \leq 6$. For $\sharp S \geq 6$, the result of Proposition 3.3 gives the answer for some posets. For a systematic analysis, we refer to the forthcoming work [2].

For $\sharp S=4$ the two relevant poset-structure are the diamond and the bowtie. Their Hasse-Diagram are, in Figure $1, \mathcal{S}_{1}$ and $\mathcal{S}_{4}$ respectively, without the node $w$. For those two posets, the computation procedure gives us that $\mathcal{G}_{\text {mon }}=\mathcal{G}_{\text {c.mon }}$ holds. Note that this result is known to be false in discrete-time, see for instance examples 1.1 and 4.5 in [3].

For $\sharp S=5$, we can then state:

Proposition 3.1 The only posets $S$ with $\sharp S \leq 5$ such that (5) does not hold are those whose HasseDiagram are presented in Figure 1.
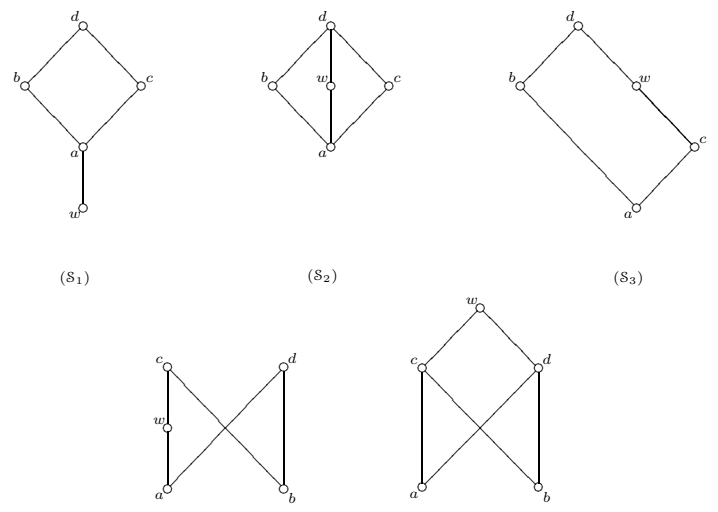

$\left(S_{2}\right)$

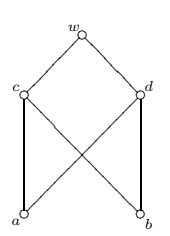

$\left(s_{3}\right)$

$\left(s_{4}\right)$

$\left(s_{5}\right)$

Figure 1. The five-points posets, for which there is no equivalence between the two notions of monotonicity.
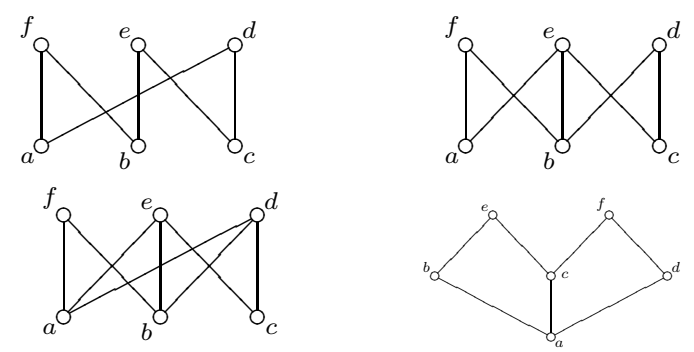

Figure 2. Some characteristic six-points posets, where there is no equivalence between the two notions of monotonicity.

We studied all five-points posets which are not linearly totally ordered. For the posets previously mentioned, we found extremal rays $L=\left(L_{x, y}\right)_{(x, y) \in S_{2}}$ of $\mathcal{G}_{c . m o n}$ which are not in $\mathcal{G}_{\text {mon }}$. One example for each poset is given in the figure 4 . The non-mentioned components are equal to 0 .

Furthermore we briefly mention the following result, whose proof is to be found in [2].

Definition 3.2 A subset $S^{\prime}$ of $S$ is said to be an (induced) subposet if for all $x, y \in S^{\prime}, x \leq y$ in $S^{\prime}$ is equivalent to $x \leq y$ in $S$. 
Proposition 3.3 If a poset $S$ admits as subposet a poset $S^{\prime}$, whose Hasse-Diagram is presented in the figures 1 and 2 then the monotonicity equivalence (i.e. the equality (5)) fails in $S$ as well.

For instance, the poset of figure 3 admits the poset $\mathcal{S}_{4}$ as subposet (consider it without the node $c$ ) and as consequence the equivalence of the two monotonicity does not hold on it.

Proposition 3.4 For $\sharp S=6$, the posets where the equivalence fails are either the ones of figure 2 or the ones which admits as subposet a poset of figure 1 .

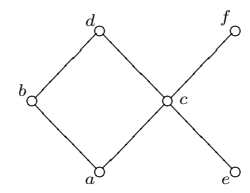

Figure 3. Six-point poset, where there is no equivalence, since it admits $\mathcal{S}_{4}$ as subposet.

$$
\begin{aligned}
& \text { on } \mathcal{S}_{1}, L_{1}: L_{a, w}=L_{b, w}=L_{c, w}=L_{d, b}=L_{d, c}=1 \\
& \text { on } \mathcal{S}_{2}, L_{2}: L_{a, b}=L_{d, b}=L_{w, b}=L_{c, d}=L_{c, a}=1 \\
& \text { on } \mathcal{S}_{3}, L_{3}: L_{d, c}=L_{b, c}=L_{a, c}=L_{w, a}=L_{c, a}=1 \\
& \text { on } \mathcal{S}_{4}, L_{4}: L_{a, b}=L_{w, b}=L_{c, d}=L_{d, b}=L_{b, d}=1 \\
& \text { on } \mathcal{S}_{5}, L_{5}: L_{w, c}=L_{w, d}=L_{c, a}=L_{d, a}=L_{b, a}=1
\end{aligned}
$$

Figure 4. Examples of monotone generators which are not complete monotone.

\section{Acknowledgements}

P.-Y. Louis thanks the Mathematics Department of the University of Padova for financing stays at Padova.

\section{References}

[1] cdd/cdd+ software, 1995-2004. available at http://www.cs.mcgill.ca/ fukuda/soft/cdd_home/cdd.html.

[2] P. Dai Pra, P.-Y. Louis, and I. Minelli. Complete monotone coupling for markov processes. in preparation.

[3] J. A. Fill and M. Machida. Stochastic monotonicity and realizable monotonicity. Ann. Probab., 29(2):938-978, 2001.

[4] K. Fukuda and A. Prodon. Double description method revisited. In Combinatorics and computer science (Brest, 1995), volume 1120 of Lecture Notes in Comput. Sci., pages 91-111. Springer, Berlin, 1996.

[5] Gap software - groups, algorithms, programming - a system for computational discrete algebra, 1986-2006. available at http://www. gap-system. org/ gap/.

[6] W. A. Massey. Stochastic orderings for Markov processes on partially ordered spaces. Math. Oper. Res., 12(2):350-367, 1987.

[7] J.G. Propp and D.B. Wilson. Exact sampling with coupled Markov chains and applications to statistical mechanics. Random Structures and Algorithms, 9(1\&2):223-252, 1996.

[8] Scilab software, 1989-2006. Copyright (C) INRIA ENPC, Scilab is a trademark of INRIA, available at http://www. scilab.org/. 